EPJ Web of Conferences 93,01019 (2015)

DOI: $10.1051 /$ epjconf/20159301019

(C) Owned by the authors, published by EDP Sciences, 2015

\title{
Isospin transfer modes in exotic nuclei
}

\author{
Elena Litvinova ${ }^{1,2, a}$ \\ ${ }^{1}$ Department of Physics, Western Michigan University, Kalamazoo, MI 49008-5252, USA \\ ${ }^{2}$ National Superconducting Cyclotron Laboratory, Michigan State University, East Lansing, MI 48824-1321, USA
}

\begin{abstract}
This work presents an approach to nuclear spin-isospin response, which is capable of describing the overall strength distribution up to high excitation energies, including the fine structure of the low-lying strength, and resolving the long-standing quenching problem. The model is a covariant realization of the nuclear field theory and based on the self-consistent extensions of the covariant energy density functional (CEDF) theory. Results of the recent calculations for spin-isospin response of ordinary and exotic medium-mass nuclei are presented and discussed.
\end{abstract}

\section{Introduction}

The spin-isospin response associated with finite spin and isospin transfer is one of the most important properties of nuclei. This type of response provides information about a variety of weak interaction processes, such as ordinary and double beta-decay, electron capture, neutrino capture and scattering on nuclei and in stars. Lately, the models, which are commonly used to describe nuclear spin-isospin response, namely Quasiparticle Random Phase Approximation and Shell Model, have advanced considerably. However, a self-consistent approach, which can simultaneously reproduce data on the overall strength distribution up to high excitation energy, quenching, and on the fine structure of the low-lying strength, is still a challenge.

This paper presents the spin-isospin version of the relativistic time blocking approximation (RTBA) as a model, which can pretend to account for all these effects. The spin-isospin RTBA (or proton-neutron RTBA, pn-RTBA) has been developed and applied for the first time to the spin-dipole resonance (SDR) in Ref. [1] and, recently, to the Gamow-Teller resonance (GTR) in Ref. [2]. Here, this approach is discussed in light of its ability to describe unstable nuclear systems and to resolve the long-standing problem of quenching of the GTR.

\section{Nuclear spin-isospin response: damping effects}

The RTBA for the spin-isospin channel is based on the extended CEDF theory $[3,4]$ and constructed similarly to the $\mathrm{R}(\mathrm{Q}) \mathrm{TBA}$ for the neutral channel developed earlier $[5,6]$, its non-relativistic version [7, 8] and applications [9-11]. The only limitation so far is that the spin-isospin RTBA does not include pairing correlations, but such a generalization is straightforward and well underway. The calculations presented below are performed in the following three steps: (i) a relativistic mean field (RMF) solution is obtained by minimization of the covariant density functional with NL3 parametrization [12], (ii) phonon spectrum and coupling vertices for the phonons with $J^{\pi}=$ $2^{+}, 3^{-}, 4^{+}, 5^{-}, 6^{+}$are obtained by the self-consistent relativistic RPA (RRPA) [13] and (iii) the Bethe-Salpeter equation is solved for the proton-neutron response function:

$$
R(\omega)=\tilde{R}^{0}(\omega)+\tilde{R}^{0}(\omega) W(\omega) R(\omega) .
$$

Here $\tilde{R}^{0}(\omega)$ is the propagator of two uncorrelated proton and neutron quasiparticles in the static mean field and the integral part contains the in-medium nucleon-nucleon interaction $W(\omega)$. The active channels of the two-body interaction $W(\omega)$ allowing for spin-flip and isospin-flip include the following static terms and the term depending on the frequency $\omega$ :

$$
W(\omega)=V_{\rho}+V_{\pi}+V_{\delta \pi}+\Phi(\omega)-\Phi(0) .
$$

$V_{\rho}$ and $V_{\pi}$ are the finite-range $\rho$-meson and the $\pi$-meson exchange interactions, respectively. They are derived from the covariant energy density functional and read [14]:

$$
\begin{array}{r}
V_{\rho}(1,2)=g_{\rho}^{2} \vec{\tau}_{1} \vec{\tau}_{2}\left(\beta \gamma^{\mu}\right)_{1}\left(\beta \gamma_{\mu}\right)_{2} D_{\rho}\left(\mathbf{r}_{1}, \mathbf{r}_{2}\right), \\
V_{\pi}(1,2)=-\left(\frac{f_{\pi}}{m_{\pi}}\right)^{2} \vec{\tau}_{1} \vec{\tau}_{2}\left(\boldsymbol{\Sigma}_{1} \nabla_{1}\right)\left(\boldsymbol{\Sigma}_{2} \nabla_{2}\right) D_{\pi}\left(\mathbf{r}_{1}, \mathbf{r}_{2}\right),
\end{array}
$$

where $g_{\rho}$ and $f_{\pi}$ are the coupling strengths, $D_{\rho}$ and $D_{\pi}$ are the meson propagators and $\boldsymbol{\Sigma}$ is the generalized Pauli matrix [14]. The Landau-Migdal term $V_{\delta \pi}$ is the contact part of the nucleon-nucleon interaction responsible for the short-range repulsion:

$$
V_{\delta \pi}(1,2)=g^{\prime}\left(\frac{f_{\pi}}{m_{\pi}}\right)^{2} \vec{\tau}_{1} \vec{\tau}_{2} \boldsymbol{\Sigma}_{1} \boldsymbol{\Sigma}_{2} \delta\left(\mathbf{r}_{1}-\mathbf{r}_{2}\right)
$$

a e-mail: elena.litvinova@wmich.edu 
where the parameter $g^{\prime}=0.6$ is adjusted to reproduce experimental data on the excitation energies of the GamowTeller resonance in ${ }^{208} \mathrm{~Pb}$ and kept fixed in the calculations for other nuclei, relying on the results obtained in Ref. [14] within the relativistic QRPA. The amplitude $\Phi(\omega)$ describes the coupling of the nucleons to vibrations generated by the coherent nucleonic oscillations. In the time blocking approximation it has the following operator form:

$$
\Phi(\omega)=\sum_{\mu, \eta} g_{\mu}^{(\eta) \dagger} \tilde{R}^{0(\eta)}\left(\omega-\eta \omega_{\mu}\right) g_{\mu}^{(\eta)},
$$

where the index $\mu$ numerates vibrational modes (phonons) with frequencies $\omega_{\mu}$ and generalized particle-vibration coupling (PVC) amplitude matrices $g_{\mu}^{(\eta)}$, and the index $\eta= \pm 1$ denotes forward and backward components, in full analogy with the neutral-channel case $[5,6]$. This amplitude is responsible for the spreading mechanism caused by the coupling between the $\mathrm{ph}$ and $\mathrm{ph} \otimes$ phonon configurations. In the calculations presented below, the phonon space is truncated by the angular momenta of the phonons at $J^{\pi}=6^{+}$and by their frequencies at $15 \mathrm{MeV}$. The $\mathrm{ph} \otimes$ phonon configurations are included up to $30 \mathrm{MeV}$ of the excitation energy. The truncation is justified by the subtraction of the term $\Phi(0)$ in Eq. (2). This subtraction removes double counting of the PVC effects from the residual interaction, guarantees the stability of the solutions for the response function and provides faster convergence of the renormalized PVC amplitude $\Phi(\omega)-\Phi(0)$ with respect to the phonon angular momenta and frequencies.

The strength function $S^{P}(\omega)$ gives the spectral distribution of the nuclear response for a particular external field $P$ expressed by the Gamow-Teller lowering operator:

$$
\begin{array}{r}
S^{P}(\omega)=-\frac{1}{\pi} \operatorname{Im}\left\langle P^{\dagger} R(E+i \Delta) P\right\rangle, \quad \omega=E+i \Delta, \\
P_{G T_{-}}=\sum_{i=1}^{A} \tau_{-}^{(i)} \boldsymbol{\Sigma}_{i} .
\end{array}
$$

The finite imaginary part of the frequency argument is typically set equal to the energy resolution of the corresponding experimental data, in order to make a correct comparison. In Figure 1(a,b), we show the results for the GTR in ${ }^{208} \mathrm{~Pb}$ obtained within the RRPA (without the last two terms in Eq. (2)) and RTBA (with the full interaction (2)), compared to the data of Ref. [15]. The RRPA calculation produces a strength with the major peak at 16.5 $\mathrm{MeV}$ and a low-energy peak structure around $10 \mathrm{MeV}$. The exact Ikeda sum rule is accommodated within the model space of pn-configurations between $-1800 \mathrm{MeV}$ and 100 $\mathrm{MeV}$, so that $8 \%$ of the $B\left(G T_{-}\right)$is at large negative energies because of the transitions to the Dirac sea [14]. While the RRPA does not account for spreading effects, within the RTBA the GTR acquires the spreading width because of the coupling between the ph and $\mathrm{ph} \otimes$ phonon configurations. Thus, the additional $5 \%$ of the sum rule goes above the considered energy region, while the total $B\left(G T_{+}\right)$is equal to 0.34 . Comparison to data shows that the spreading effects which are taken into account in the
RTBA are reproduced very well. The overall strength is visibly quenched compared to the RRPA one, however, the cumulative sum shows that the experimental integral strength is still smaller than the RTBA one. A study of Ref. [16] has revealed that the rest of the observed quenching can come from the finite momentum transfer, which is not included in the present calculations. Figure 1(c-f) shows the GTR in neutron-rich ${ }^{78} \mathrm{Ni}$, whose properties are interesting to study because of their astrophysical importance. The damping caused by the particle-phonon coupling affects not only the giant resonance, but also the low-lying strength, whose portion below the beta-decay window $Q_{\beta}$ defines the lifetime. Within the RTBA, this portion becomes considerably smaller than in the RRPA and allows for direct determination of the half-life without introducing an artificial quenching factor.

\section{Model space and quenching of the GTR}

In the context of the quenching problem the size of the basis spanning the ph $\otimes$ phonon configurations plays an important role. In the numerical calculations, the energy window containing these configurations is that, in which the amplitude of Eq. (6) has non-zero matrix elements. The truncation of this energy window is just a technical necessity and varies depending on the available computing resources. In the case of ${ }^{208} \mathrm{~Pb}$ it turns out that almost the whole effect of coupling to the ph $\otimes$ phonon configurations appears within $30 \mathrm{MeV}$ of the excitation energy. An extension of the $p h \otimes$ phonon configuration window to $60 \mathrm{MeV}$ does not influence the results. The particle-hole proton-neutron configurations are included, however, up to $100 \mathrm{MeV}$, so that about 10 units of the Ikeda sum rule are absorbed by the GT strength between 25 and $100 \mathrm{MeV}$. Further increase of the ph-basis size would spread the GT strength wider, but very slowly, so that, although effectively there is no need to extend the basis beyond 100 $\mathrm{MeV}$, in principle, it is not clear how to justify the cutoff rigorously. Overall, the extension of the ph $\otimes$ phonon window involves the inversion of larger matrices, but nevertheless, in the present formulation this window is considerably larger than that, for instance, in typical shell-model calculations. Moreover, the present approach allows an investigation of the convergence of the results with respect to this window. As discussed above, in the case of ${ }^{208} \mathrm{~Pb}$ the value of $30 \mathrm{MeV}$ can be taken as the cutoff energy for the $p h \otimes$ phonon configurations.

As the next example, a neutron deficient $\mathrm{N}=\mathrm{Z}$ nucleus ${ }^{28} \mathrm{Si}$ is chosen. Being a considerably lighter than ${ }^{208} \mathrm{~Pb}$, ${ }^{28} \mathrm{Si}$ has much lower level density, so that one can expect a higher saturation energy for the $\mathrm{ph} \otimes$ phonon configurations. Indeed, the pn-RTBA calculations confirm this expectation which is illustrated in Figure 2. In this calculations the $p h \otimes$ phonon configurations are accomodated within $90 \mathrm{MeV}$ energy interval, which is close to that, where $1 \mathrm{p} 1 \mathrm{~h}$ pn-RRPA configurations are included (100 $\mathrm{MeV}$ ). One can see from the left panel of Figure 2, that a part of the GT_ strength appears at large negative energies, which is the typical feature of relativistic approaches using 

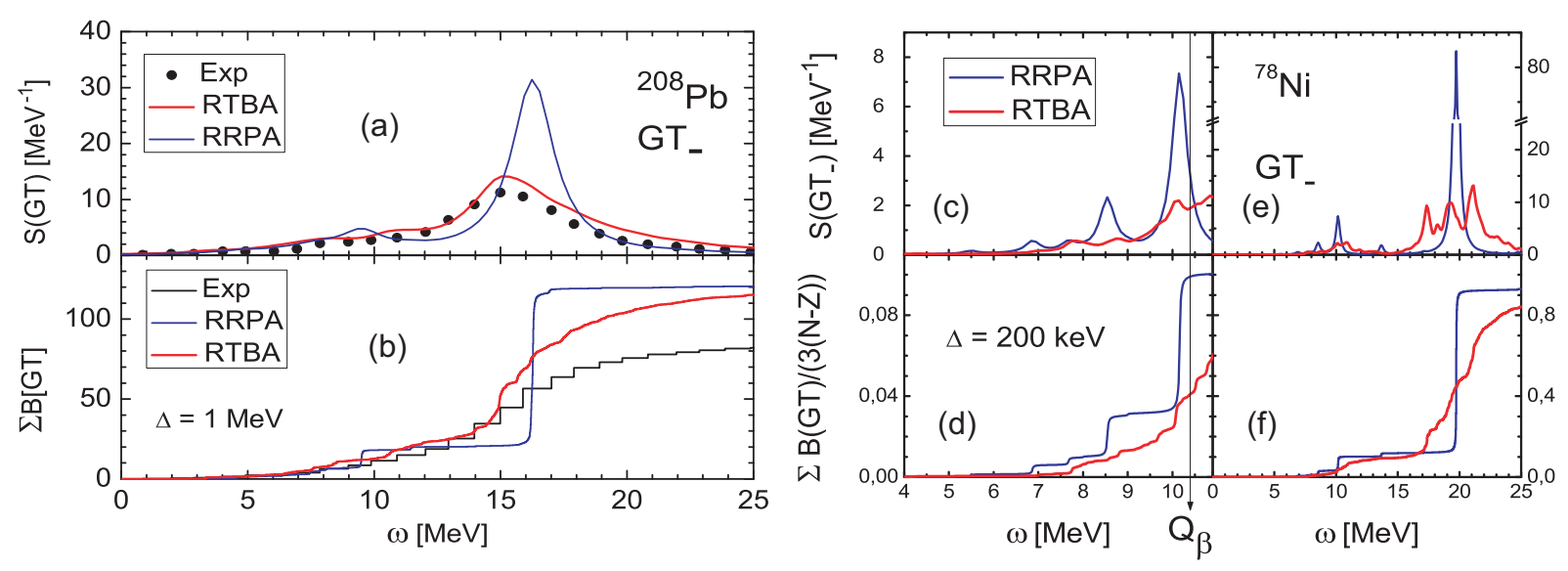

Figure 1. Theoretical and experimental Gamow-Teller strength distributions in ${ }^{208} \mathrm{~Pb}$ (a) and their cumulative sums (b). GTR strength in the neutron-rich ${ }^{78} \mathrm{Ni}$ : the low-energy portion of the strength relevant for the beta-decay lifetime (c), its cumulative sum (d), the overall strength distribution up to $25 \mathrm{MeV}$ (e) and its cumulative sum (f). $Q_{\beta}$ shows the beta-decay window.
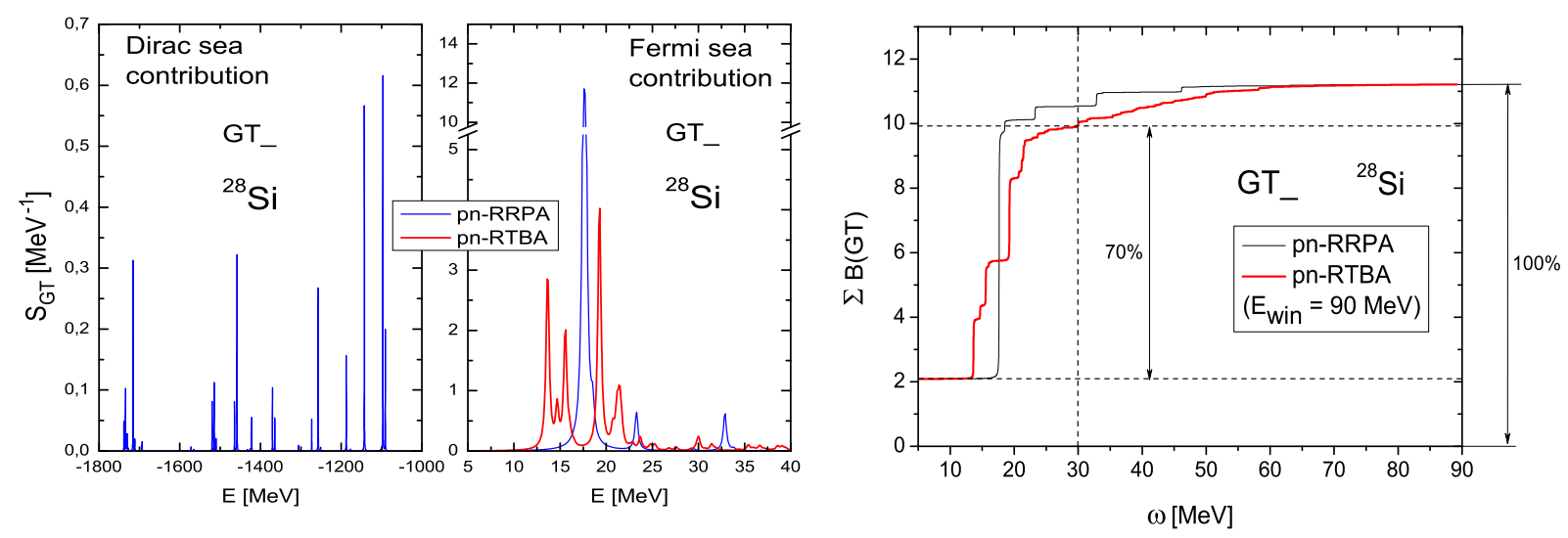

Figure 2. Gamow-Teller strength distribution for ${ }^{28} \mathrm{Si}$. Left panel: negative-energy part of the strength originated from the protonantineutron transitions to the Dirac sea. Middle: positive-energy part of the GTR strength in pn-RRPA and in pn-RTBA. Right: Running sums for the pn-RRPA and pn-RTBA strength distributions.

no-sea approximation. In the nucleon sector, the cumula-

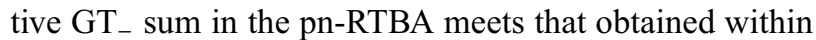
the pn-RRPA at $E_{c} \sim 60 \mathrm{MeV}$, which is sufficiently smaller than the pn-RTBA window. Thus, the GT - strength between 0 and $30 \mathrm{MeV}$ (the energy region which is usually associated with the GTR) amounts $70 \%$ of the total GT _ strength. This characterizes the ability of the pnRTBA to explain the quenching, which is observed experimentally in restricted energy intervals, by the damping through $1 \mathrm{p} 1 \mathrm{~h} \otimes$ phonon configurations. The $\mathrm{GT}_{+}$strength distribution (not shown) in this nucleus looks very similar although not exactly the same as the $G T_{-}$. The unique feature of $\mathrm{N}=\mathrm{Z}$ nuclei is that the Ikeda sum rule

$$
S\left(G T_{-}\right)-S\left(G T_{+}\right)=3(N-Z)
$$

is exactly zero which allows a stringent test of the approach and of the numerical implementation. Although the sum rule gives no values for the $\mathrm{GT}_{+}$and $\mathrm{GT}_{-}$distributions separately, it is fulfilled in the present calculations for the GTR in ${ }^{28} \mathrm{Si}$ with $\sim 0.2 \%$ accuracy, with respect to the total $\mathrm{GT}_{+}$or GT_ strength.

\section{Summary and outlook}

This paper presents selected results obtained in the covariant nuclear field theory, recently developed for the nuclear response in the isospin channel. The one-boson exchange nucleon-nucleon interaction includes effective mesons and the emergent vibrational quanta which account for the time dependence of both meson and nucleonic fields. The performance of the approach is illustrated in the calculations for Gamow-Teller strength in ${ }^{208} \mathrm{~Pb},{ }^{78} \mathrm{Ni}$ and ${ }^{28} \mathrm{Si}$. It is shown how the fragmentation of the strength appears due to the coupling to vibrations, in addition to Landau damping. The quenching of the GTR because of the spreading to high energies and its connection with the completeness of the model space are discussed. Further advancements of the pn-RTBA, such as implementation of the superfluidtype pairing and higher-order correlations, will be performed as the next steps of the model development. Experimental data on the spin-isospin strength distributions in broad energy intervals are expected from future measurements of various exotic nuclei at the rare isotope beam facilities. Such data will provide decisive arguments to 
constrain many-body coupling schemes of the R(Q)TBA as well as the underlying nuclear effective interactions.

\section{Acknowledgements}

Fruitful discussions with P. Ring, V. Tselyaev, T. Marketin, R. Zegers, A. Brown, and D.-L. Fang are gratefully acknowledged. This work was supported by the NSF grants PHY-1204486 nd PHY-1404343.

\section{References}

[1] T. Marketin, E. Litvinova, D. Vretenar, and P. Ring, Phys. Lett. B 706, 477 (2012)

[2] E. Litvinova, B.A. Brown, D.-L. Fang, T. Marketin, and R. G. T. Zegers, Phys. Lett. B 730, 307 (2014)

[3] E. Litvinova and P. Ring, Phys. Rev. C 73, 044328 (2006)

[4] E. Litvinova, Phys. Rev. C 85, 021303(R) (2012)

[5] E. Litvinova, P. Ring, and V. Tselyaev, Phys. Rev. C 75, 064308 (2007)

[6] E. Litvinova, P. Ring, and V. Tselyaev, Phys. Rev. C 78, $014312(2008)$
[7] E.V. Litvinova and V.I. Tselyaev, Phys. Rev. C 75, 054318 (2007)

[8] V. Tselyaev, J. Speth, F. Grümmer et al., Phys. Rev. C 75, 014315 (2007)

[9] J. Endres, E. Litvinova, D. Savran et al., Phys. Rev. Lett. 105, 212503 (2010)

[10] J. Endres, D. Savran, P.A. Butler et al., Phys. Rev. C 85, 064331 (2012)

[11] R. Massarczyk, R. Schwengner, F. Dönau et al., Phys. Rev. C 86, 014319 (2012)

[12] G. A. Lalazissis, J. König, and P. Ring, Phys. Rev. C 55, 540 (1997)

[13] P. Ring, Z.-Y. Ma, N. Van Giai, D. Vretenar, A. Wandelt, and L.-G. Cao, Nucl. Phys. A 694, 249 (2001)

[14] N. Paar, T. Nikšić, D. Vretenar, and P. Ring, Phys. Rev. C 69, 054303 (2004)

[15] T. Wakasa, M. Okamoto, M. Dozono, K. Hatanaka, M. Ichimura, S. Kuroita, Y. Maeda, H. Miyasako, T. Noro, T. Saito, Y. Sakemi, T. Yabe, and K. Yako, Phys. Rev. C 85, 064606 (2012)

[16] T. Marketin, G. Martínez-Pinedo, N. Paar and D. Vretenar, Phys. Rev. C 85, 054313 (2012) 